\title{
Glial Activation in the Early Stages of Brain Metastasis: TSPO as a Diagnostic Biomarker
}

\author{
Emma R. O'Brien ${ }^{1}$, Veerle Kersemans ${ }^{1}$, Matthew Tredwell ${ }^{2}$, Begona Checa ${ }^{2}$, Sébastien Serres ${ }^{1}$, Manuel Sarmiento Soto ${ }^{1}$, \\ Veronique Gouverneur ${ }^{2}$, David Leppert ${ }^{3}$, Daniel C. Anthony ${ }^{4}$, and Nicola R. Sibson ${ }^{1}$ \\ ${ }^{I}$ CR-United Kingdom/MRC Gray Institute for Radiation Oncology and Biology, Department of Oncology, University of Oxford, \\ Churchill Hospital, Oxford, United Kingdom; ${ }^{2}$ Chemistry Research Laboratory, University of Oxford, Oxford, United Kingdom; \\ ${ }^{3}$ Department of Neurology, University Hospitals Basel, Basle, Switzerland; and ${ }^{4}$ Department of Pharmacology, University of Oxford, \\ Oxford, United Kingdom
}

\begin{abstract}
Metastatic spread of cancer cells to the brain is associated with high mortality, primarily because current diagnostic tools identify only well-advanced metastases. Brain metastases have been shown to induce a robust glial response, including both astrocyte and microglial activation. On the basis of these findings, we hypothesized that this stromal response may provide a sensitive biomarker of tumor burden, in particular through the use of SPECT/PET imaging agents targeting the translocator protein (TSPO) that is upregulated on activated glia. Our goals, therefore, were first to determine the spatial and temporal profile of glial activation during early metastasis growth in vivo and second to assess the potential of the radiolabeled TSPO ligand ${ }^{123}$ I-DPA-713 for early detection of brain metastases. Methods: Metastatic mouse mammary carcinoma 4T1green fluorescent protein cells were injected either intracerebrally or intracardially into female BALB/c mice to induce brain metastases. Astrocyte and microglial activation was assessed immunohistochemically over a 28-d period, together with immunofluorescence detection of TSPO upregulation. Subsequently, SPECT imaging and autoradiography were used to determine in vivo binding of 123|-DPA-713 at metastatic sites. Results: Dynamic astrocyte and microglial activation was evident throughout the early stages of tumor growth, with the extent of astrocyte activation correlating significantly with tumor size $(P<0.0001)$. Microglial activation appeared to increase more rapidly than astrocyte activation at the earlier time points, but by later time points the extent of activation was comparable between the glial cell types. Upregulation of TSPO expression was found on both glial populations. Both autoradiographic and in vivo SPECT data showed strong positive binding of ${ }^{123}$ I-DPA-713 in the intracerebrally induced model of brain metastasis, which was significantly greater than that observed in controls $(P<0.05)$. ${ }^{123}$ I-DPA-713 binding was also evident autoradiographically in the intracardially induced model of brain metastasis but with lower sensitivity because of smaller tumor size $(\sim 100-\mu \mathrm{m}$ diameter vs. $\sim 600-\mu \mathrm{m}$ diameter in the intracerebral model). Conclusion: These data suggest that the glial response to brain metastasis may provide a sensitive biomarker of tumor burden, with a tumor detection threshold lying between 100 and $600 \mu \mathrm{m}$ in diameter. This approach could enable substantially earlier detection of brain metastases than the current clinical approach of gadolinium-enhanced MR imaging.
\end{abstract}

Received Jun. 18, 2013; revision accepted Oct. 18, 2013.

For correspondence or reprints contact: Nicola R. Sibson, Experimental Neuroimaging Group, Radiobiology Research Institute, Churchill Hospital, Oxford, OX3 7LJ, U.K.

E-mail: nicola.sibson@oncology.ox.ac.uk

Published online Jan. 16, 2014.

COPYRIGHT (c) 2014 by the Society of Nuclear Medicine and Molecular Imaging, Inc.
Key Words: astrocytes; microglia; metastasis; TSPO; SPECT imaging; mouse

J Nucl Med 2014; 55:275-280

DOI: 10.2967/jnumed.113.127449

$\mathbf{M}$ etastasis is the leading cause of cancer morbidity and mortality, and $10 \%-40 \%$ of all cancer patients are estimated to develop metastatic spread of their disease to the brain (1). However, our understanding of brain metastasis is still in its relative infancy and, in addition to understanding the pathogenic mechanisms underlying metastatic progression, there is an urgent need to identify new biomarkers for early tumor detection.

Currently, clinical detection of brain metastasis relies on gadolinium contrast-enhanced MR imaging. However, this approach is dependent on blood-brain barrier breakdown, which does not occur until relatively late in disease progression (2). As a consequence, therapy is limited and prognosis poor. Thus, the development of new imaging approaches enabling earlier detection is of critical importance. Previous work from our laboratory has demonstrated the potential for early metastasis detection based on the tumor microenvironment, specifically the activation of the endothelium and expression of cell adhesion molecules, such as vascular cellular adhesion molecule 1 (3). However, other central nervous system stromal cell types may also yield targets for diagnostic biomarkers.

Previously, a mixed glial response to brain metastases, including reactive astrocytes and microglia, has been reported both in animal models and human brain tissue. Astrocytes are the most abundant of the nonneuronal cell populations in the brain and have multiple homeostatic and metabolic roles in the central nervous system. Astrocytes also actively respond to challenges such as infection and neurodegeneration, by changing their transcriptional profile and morphology in a characteristic process of reactive astrogliosis. Reactive astrocytes have been reported around brain metastases in human postmortem tissue $(4,5)$, and there has been increasing interest in determining their significance in mouse models of brain metastasis (6-9). Nevertheless, their contribution to the progression of brain metastases remains unclear, with both pro- and antitumorigenic roles being proposed $(6,10-14)$. At the same time, activated microglia, the resident macrophages of the brain, have also been found at sites of brain metastases $(4,5,9)$, and these findings suggest that these 2 glial populations may be intimately involved in tumor progression. 
The translocator protein (TSPO), or peripheral benzodiazepine receptor, is a protein located on the outer mitochondrial membrane (15) and has multiple functions, including cholesterol import for steroid synthesis, regulation of mitochondrial metabolism, and apoptosis. Importantly, although constitutively expressed, this protein is highly upregulated on activated glia. Originally, TSPO upregulation in disease was considered to be exclusive to activated microglia, but recent studies have also demonstrated marked upregulation on reactive astrocytes $(16,17)$. Concomitantly, interest has grown in the development and use of radiolabeled compounds against TSPO for imaging neuroinflammation in central nervous system pathologies (18), including recent studies in glioma $(19,20)$.

On the basis of the glial response to brain metastases that has previously been reported, we hypothesized that TSPO-targeted diagnostic imaging agents might provide a sensitive biomarker of metastasis burden in the brain. The primary aims of the current study, therefore, were to quantitatively determine the temporal and spatial profile of astroglial and microglial activation over the early time course of metastasis pathogenesis in vivo and, subsequently, to assess the potential of reactive gliosis as an imaging biomarker for early detection of brain metastases using the TSPO-targeted SPECT imaging agent ${ }^{123}$ I- $N, N$-diethyl-2-[2-(4-hydroxy-phenyl)-5,7-dimethylpyrazolo[1,5-a]pyrimidin-3-yl]-acetamide ( ${ }^{123}$ I-DPA713) (21).

\section{MATERIALS AND METHODS}

Two mouse models of brain metastasis were used: direct tumor cell injection into the brain to establish a reproducible focal metastatic site at a known location, facilitating extended longitudinal studies with the contralateral hemisphere serving as an internal control, and hematogenous induction via intracardiac injection of tumor cells. The mouse mammary carcinoma 4T1-green fluorescent protein (GFP) cell line was used in both cases in 6- to 7-wk-old female BALB/c mice (Charles River). All procedures were performed in accordance with the U.K. Animal (Scientific Procedures) Act 1986. The supplemental materials provide a comprehensive description of methods (supplemental materials are available at http://jnm.snmjournals.org).

\section{Brain Metastasis Models}

For the intracerebral model of brain metastasis, animals were injected stereotactically in the striatum $(+0.5 \mathrm{~mm} ; 1.5 \mathrm{~mm}$ lateral; depth, -2.5 $\mathrm{mm}$ from Bregma), with either $0.5 \mu \mathrm{L}$ of saline containing $5 \times 10^{3} 4 \mathrm{~T} 1$ GFP cells or $0.5 \mu \mathrm{L}$ of vehicle (saline) alone using a pulled glass microcapillary (diameter, $75 \mu \mathrm{m}$; Clark Electromedical Instruments). At days 10, 14, 21, and 28 (4T1-GFP, $n=5$; saline, $n=3$ per time point), mice were transcardially perfused with $0.9 \%$ heparinized saline, followed by periodate lysine paraformaldehyde containing $0.025 \%$ glutaraldehyde (periodate lysine paraformaldehyde light). Tissue was embedded (Tissue-Tek; Bayer Plc), and 10- $\mu \mathrm{m}$-thick serial coronal sections were taken using a cryostat (Leica). For the intracardiac model, mice were anesthetized with $2 \%$ isoflurane in oxygen and intracardially injected in the left ventricle (via ultrasound guidance (22)) with $10^{4} 4 \mathrm{~T} 1$-GFP cells in $100 \mu \mathrm{L}$ of phosphate-buffered saline. At days $10,14,21$, and 28 after injection, animals were perfusionfixed and tissue was processed as above ( $n=5-6$ per time point).

\section{Immunohistochemistry and Immunofluorescence}

Briefly, sections were quenched in methanol with $1 \% \mathrm{H}_{2} \mathrm{O}_{2}(30 \%$ w/w). Subsequently, tissue was blocked and incubated with the relevant primary antibody overnight at $4^{\circ} \mathrm{C}$ : rabbit anti-GFAP (glial fibrilary acidic protein) for astrocytes (1:500, ZO334; Dako), goat anti-IBA1 (1:500, ab5076; Abcam), or rat anti-OX42 (1:200, MCA711; Serotec) for microglia. Primary antibody binding was detected using the relevant biotinylated secondary antibody and an $\mathrm{ABC}$ kit (both 1:100, Vector
Laboratories). Immunoreactivity was revealed using standard diaminobenzidine $\mathrm{HCl}$ histochemistry and sections counterstained with cresyl violet. Sections were scanned using the ScanScope CS system (Aperio). Areas of tumor, astrocyte activation, and microglial activation were demarcated using ImageScope software (Aperio). Fluorescent colocalization was used to determine TSPO expression in astrocytes, microglia, 4T1-GFP cells, and MDA-231Br cells. Full details of antibodies, immunohistochemistry, immunofluorescence, and image analysis are provided in the supplemental data.

\section{SPECT}

DPA713 was synthesized as previously described (23). Radiolabeling was performed following a modified literature procedure (24) and formulated in ethanol. Quality control was performed for each sample. Full details are provided in the supplemental data.

For the intracerebral model, BALB/c mice were imaged $13 \mathrm{~d}$ after intracerebral injection of either 4T1-GFP cells $(n=6)$ or saline $(n=$ 3 ), as described above. Animals were intravenously injected with 20 MBq of ${ }^{123}$ I-DPA713 $1 \mathrm{~h}$ before SPECT. Mice were anesthetized with $1.5 \%-2.0 \%$ isoflurane in air, and SPECT/CT was performed using a nanoSPECT/CT scanner (Bioscan) equipped with 9-pinhole apertures (pinhole diameter, $1 \mathrm{~mm}$ ). SPECT imaging was performed as described in the supplemental data.

For the intracardiac model, mice were imaged $21 \mathrm{~d}$ after intracardiac injection of either $1 \times 10^{4} 4 \mathrm{~T} 1$-GFP cells $(n=5)$ or saline $(n=3)$. SPECT/CT was performed as described above. A volume of interest was drawn across the whole brain using the CT images as an anatomic reference. Average intracerebral activity $(\mathrm{kBq})$ over the volume of interest was normalized to average extracranial activity in an extracranial volume of interest across the base of the skull, using the $\mathrm{CT}$ images as the anatomic reference.

After SPECT imaging, animals were transcardially perfused with heparinized saline. Brains were removed and fresh-frozen in isopentane and dry ice and transferred to a cryostat at $-20^{\circ} \mathrm{C}$. Coronal sections $(20$ $\mu \mathrm{m}$ thick) were cut, with serial sections taken for immunohistochemistry and autoradiography. For autoradiography, slides were placed on Super Resolution Phosphor screens (Perkin Elmer). Screens were held in autoradiography cassettes (Fisher Biotech) for $36 \mathrm{~h}$ at $4^{\circ} \mathrm{C}$. Subsequently, the phosphor plate was read with Cyclone Plus using OptiQuant software (Perkin Elmer). For immunohistochemistry, slides were postfixed in $4 \%$ paraformaldehyde (PFA) for $30 \mathrm{~min}$ at $4{ }^{\circ} \mathrm{C}$ and then underwent immunohistochemical staining as described above. Mean intensity was calculated across 4 striatal sections in ImageJ (National Institutes of Health), and relative change in compound binding was calculated (ipsilateral - contralateral/contralateral $\times 100$ ).

\section{Statistical Analysis}

All data were analyzed in Prism (version 5.0; GraphPad Software), with bar graphs displaying mean \pm SEM. When 2 groups were compared, a 2-tailed, unpaired Student $t$ test was used. For multiple groups, 1-way or 2-way ANOVAs were used, with Tukey post hoc or Bonferroni post hoc tests used, respectively.

\section{RESULTS}

\section{Temporal and Spatial Profile of Reactive Gliosis in Brain Metastasis}

For the intracerebral model, in all cases brain metastases were associated with a surrounding area of activated astrocytes, which persisted across the 28-d time course (Fig. 1A). A significant positive correlation was found between tumor volume and the volume of associated astrocyte activation (Fig. 1B), and the ratio of astrocyte activation to tumor volume remained constant throughout (Fig. 1C). Astrocyte activation was also observed in saline-injected animals, 


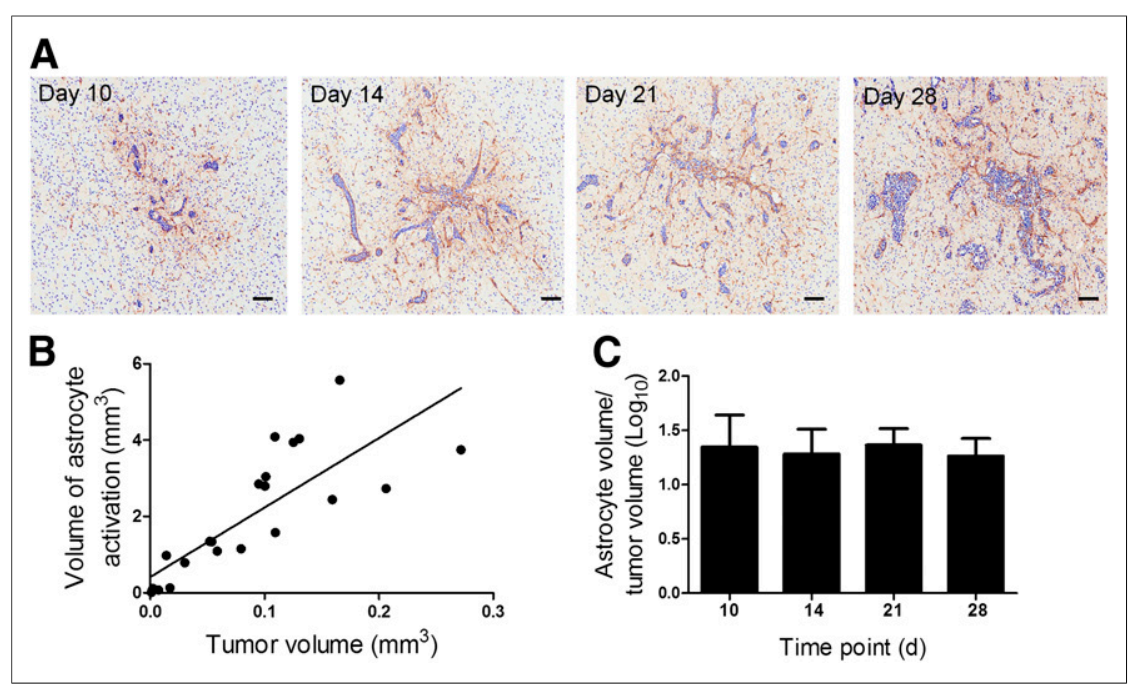

FIGURE 1. (A) Photomicrographs of brain sections from animals at days 10, 14, 21, and 28 after intracerebral injection of 4T1-GFP cells. Reactive astrocytes were identified by GFAP immunoreactivity (brown stain), and sections were counterstained with cresyl violet. Scale bar $=100 \mu \mathrm{m}$. (B) Significant positive correlation was found between volume of astrocyte activation and tumor volume $\left(y=18.2 x+0.415 ; r^{2}=0.657 ; P<0.0001\right)$. (C) No significant differences were found in ratio of astrocyte to tumor volume over time ( $n=4-5$ per group).

but this was significantly less than in 4T1-GFP-injected animals and decreased rapidly across the time course (Supplemental Fig. 1).

Similarly, in the intracardiac model, brain metastases were associated with a surrounding area of activated astrocytes, which again persisted across the 28-d time course (Fig. 2A). A significant positive correlation was found between tumor area and the area of astrocyte activation across time (Fig. 2B). However, in this case, the ratio of astrocyte activation to tumor area also increased significantly $(P<0.05$; Fig. $2 C)$, indicating a greater increase in the area of astrocyte activation than tumor growth.

Microglial activation was also evident at the sites of brain metastases in both the intracerebral and the intracardiac models but showed greater infiltration of the metastasis core than the reactive astrocytes (Fig. 2D). For the intracerebral model, the extent of microglial activation was comparable to the extent of astrocyte activation (data not shown). In the intracardiac model, a significantly greater area of microglial activation than astrocyte activation was found at days 10 and 14 after 4T1-GFP injection (Fig. 2D; $P<$ 0.001 ); however, no significant difference was found at day 28 .

\section{TSPO Upregulation on Tumor Cells, Astrocytes, and Microglia}

TSPO expression was found to colocalize with both astrocytes and microglial cells in both the intracerebral model (day 14; Fig. $3 \mathrm{~A}$ ) and the intracardiac model (day 21; Fig. 3B). Expression of TSPO in the 4T1-GFP cells was shown to be low in vitro, as compared with the positive control MDA231BR-GFP cells (Supplemental Fig. 2), and colocalization between TSPO and 4T1-GFP cells was not observed in vivo. Although colocalization of TSPO with astrocytes and microglia was evident in both models, the degree of TSPO expression in the different glial cell types appeared to vary between the models. In the intracerebral model, astrocytes showed more marked TSPO expression than microglia. Conversely, in the intracardiac model TSPO expression in astrocytes was largely restricted to the tumor periphery, whereas microglia displayed more robust expression than in the intracerebral model.
123|-DPA713 Imaging of

Tumor-Associated Gliosis in

Intracerebral Model

To determine whether the radiolabeled TSPO ligand could have potential for early metastasis detection, on the basis of the mixed glial response to tumor growth, the agent ${ }^{123}$ I-DPA713 was tested in vivo. In the first instance, the intracerebral model was used because of the known location of metastatic foci, and day 13 after induction was chosen because of the large area of astrocyte and microglial activation and concomitant TSPO expression present. After intravenous injection of ${ }^{123}$ I-DPA713, areas of increased signal intensity on SPECT images were clearly evident in the hemisphere containing metastases whereas no signal changes were evident in either the contralateral control hemisphere or the saline-injected control mice (Fig. 4A). The areas of signal increase corresponded to the metastasis-bearing region, and the in vivo observations were confirmed with ex vivo autoradiography (Fig. 4B). Quantitatively, a significant increase in ${ }^{123}$ I-DPA713 binding was found in the tumor-bearing striatum, compared with saline-injected controls from both the in vivo SPECT $(P<0.05$; Fig. 4C) and the ex vivo autoradiography $(P<0.05$; Fig. 4D) data.

Coregistration of autoradiographic and immunohistochemical images confirmed that ${ }^{123}$ I-DPA713 binding was limited to areas of tumor-associated glial activation and correlated spatially with both microglial and astrocytic activation (Supplemental Fig. 3). In all cases, the area of strongest ${ }^{123}$ I-DPA713 signal appeared to correlate with the tumor foci, which encompassed a dense microglial infiltrate and a border of astrocyte activation (Supplemental Fig. 3).

\section{3|-DPA713 Imaging of Tumor-Associated Gliosis in Intracardiac Model}

Subsequently, ${ }^{123}$ I-DPA713 was tested in the intracardiac model. In this case, animals were imaged $21 \mathrm{~d}$ after induction at a point where metastases are on the order of $100 \mu \mathrm{m}$ in diameter and robust glial activation is evident. As for the intracerebral model, ${ }^{123}$ I-DPA713 enabled autoradiographic detection of glial activation associated with brain metastases in the intracardiac model, and regions of increased signal intensity correlated with tumor-associated gliosis identified immunohistochemically (Fig. 5). In contrast, using in vivo SPECT, individual metastases were not readily detectable in this model. Across the entire brain, a trend toward an increase in specific activity in 4T1-GFP-injected mice was evident but did not reach significance.

\section{DISCUSSION}

Probing the tumor microenvironment as a biomarker for metastatic burden may provide a novel approach for the early detection of brain metastases. In this study, we have determined the temporal and spatial profile of glial activation over the early time course of metastasis pathogenesis in vivo. We have demonstrated both astrocyte and microglial activation from early in tumor development. Subsequent SPECT imaging targeting reactive glia via upregulation of TSPO indicated that this glial response could be used to detect 


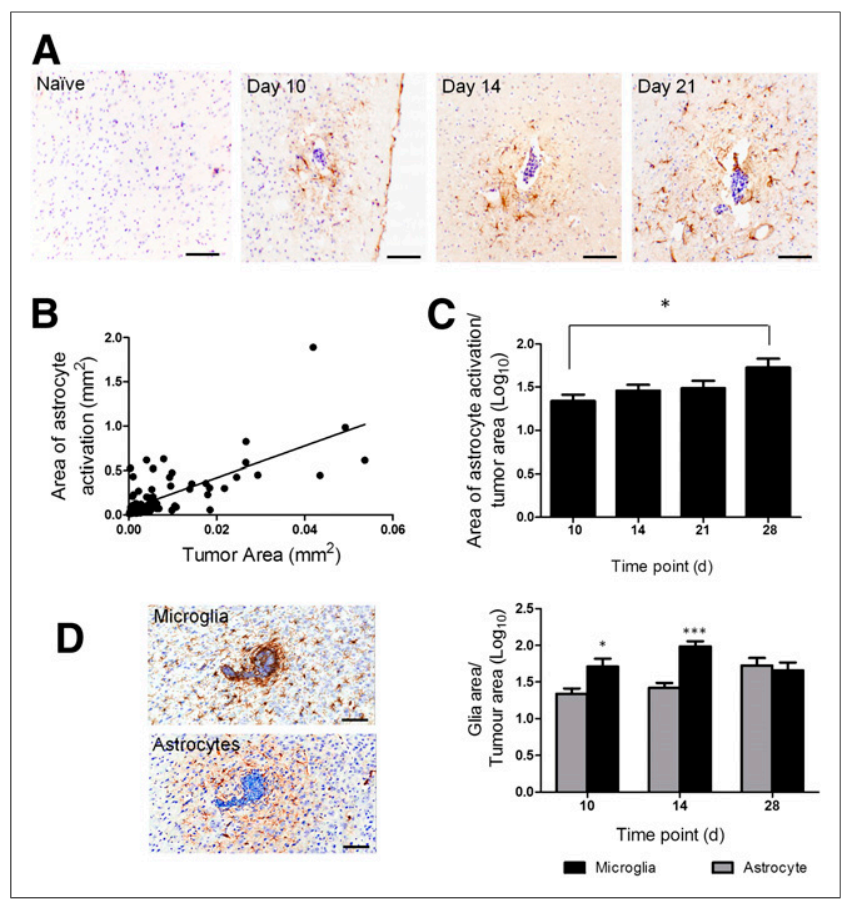

FIGURE 2. (A) Photomicrographs of brain sections from a naive BALB/C mouse and at days 10, 14, and 28 after intracardiac injection of 4T1-GFP cells. Reactive astrocytes were identified by GFAP immunoreactivity (brown stain), and sections were counterstained with cresyl violet. Scale bar $=100 \mu \mathrm{m}$. (B) Significant positive correlation was found between extent of astrocyte activation and tumor area $\left(y=11 x+0.006 ; r^{2}=0.6\right.$; $P<0.0001$ ). (C) Significant increase in ratio of astrocyte to tumor area was found over time $\left({ }^{*} P<0.05, n=5-6\right.$ per group). (D) Photomicrographs comparing microglial infiltration of metastases, detected by lba-1 immunoreactivity, with astrocyte activation (brown stain in each case). Graph shows quantitation of microglial (black bars) and astrocyte (gray bars) activation at days 10 (tumor number $=16), 14(n=39)$, and $28(n=7)$. ${ }^{\star} P<0.05 .{ }^{* \star} P<0.0001$.

metastatic growth in the brain and notably provides a greater target area for detection than the metastases themselves. These findings suggest that TSPO-targeted PET or SPECT may provide a useful imaging biomarker of early metastasis growth in the brain based on the tumor-associated glial activation.

Several studies, primarily in vitro, suggest that astrocytes may have both anti- and protumorigenic roles. On the one hand, astrocytes produce tumoricidal nitric oxide (10) and recruit systemic immune cells that may exhibit tumoricidal activity such as cytotoxic T cells (11). Conversely, astrocyte-tumor cell cocultures suggest that astrocytes may activate tumor cell proliferative pathways (12) via secretion of soluble factors such as tumor necrosisfactor (TNF) and IL-6 (13). Moreover, coculture with astrocytes has been shown to induce reprogramming of the tumor cell transcriptome, mirroring transcriptional changes observed in vivo (25) and thus increasing both invasiveness (6) and chemoresistance $(26,27)$. At the same time, microglial activation is also known to be a feature of the brain metastatic microenvironment $(4,5,28,29)$ and, as with astrogliosis, studies suggest both anti- and protumorigenic roles for microglia. For example, microglial-induced tumor cytotoxicity has been demonstrated in vitro $(30,31)$, but these cells have also been shown to facilitate metastatic colonization (29).

Here, we have shown that brain metastases are closely associated with both astrocyte and microglial activation in vivo, early in disease progression and consistently throughout the 28-d time course studied. In the case of astrocytes, these findings are in agreement with those of Fitzgerald et al. (8), who have shown that brain metastases are associated with reactive gliosis at 14 and 28 $\mathrm{d}$ after induction in a xenograft model. Here, we have recapitulated and extended these findings in a syngeneic and immunocompetent mouse model of brain metastasis, both through hematogenous dissemination of tumor cells and through direct introduction into the brain. In both cases, the presence of reactive astrocytes was observed from the earliest time points studied and was considerably greater than the tumor area itself $(\sim 25$-fold for the intracerebral model, $\sim 45$-fold for the intracardiac model). Quantitatively, the area of astrogliosis correlated positively with tumor growth. Similar positive correlations between astrogliosis and tumor burden have been demonstrated in an in vivo model of lung cancer brain metastasis (13), although at a single time point only. In both models used here, reactive astrocytes did not appear to form a fixed gliotic scar around the tumor, as is often seen surrounding an ischemic lesion, for example. Rather, the ratio of astrogliosis to tumor size either remained constant (intracerebral model) or increased with time (intracardiac model). These findings suggest that the tumor continues to release astrocyte-activating factors as it grows, such that the gliosis neither resolves nor walls off the tumor in a static rim, and are in accord with in vitro work indicating that tumor-induced astrocyte activation is not contact-dependent (13).

Again in accord with previous literature, activated microglia were detected in both models in the current study. In the intracerebral model, the extent of microglial activation was comparable to that of astrocyte activation at the 2 time points studied. In the intracardiac model, the microglial response appeared to be more robust than astrocyte activation over the early stages, with microglial activation encompassing an area approximately 80-fold greater than the tumor area at days 10 and 14 and subsequently plateauing, whereas astrocyte activation increased gradually across the time course.

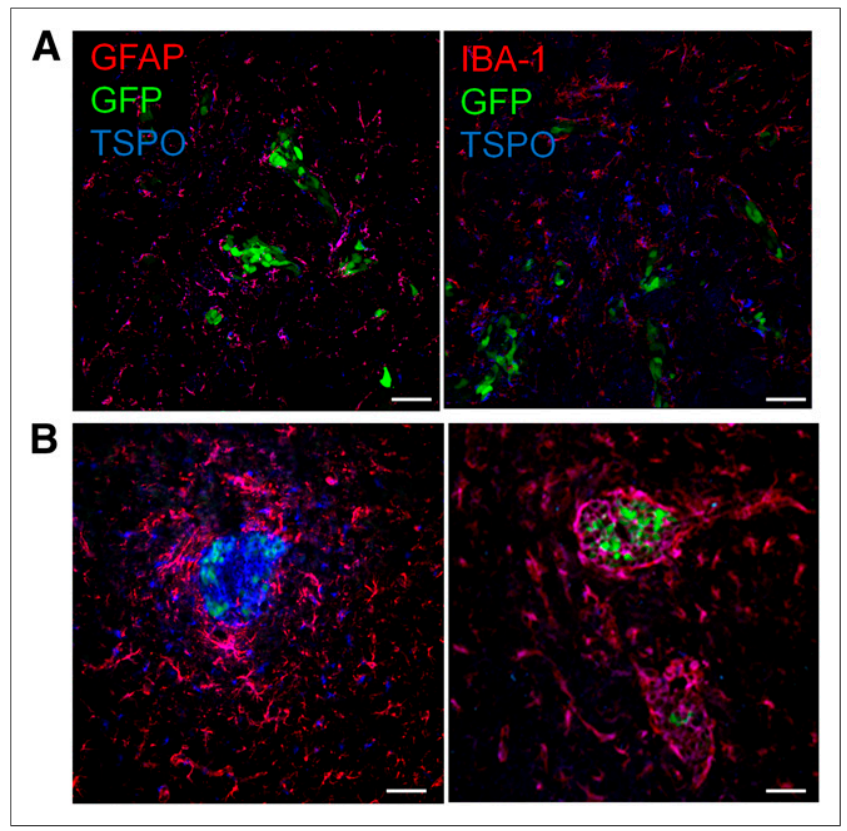

FIGURE 3. Confocal microscopy images obtained from mouse brain $14 \mathrm{~d}$ after intrastriatal injection of 4T1-GFP cells (A) or $21 \mathrm{~d}$ after intracardiac injection (B) demonstrating TSPO colocalization with either astrocyte reactivity (GFAP) surrounding metastases or reactive microglia in tumor periphery (IBA-1). Scale bar $=50 \mu \mathrm{m}$. 


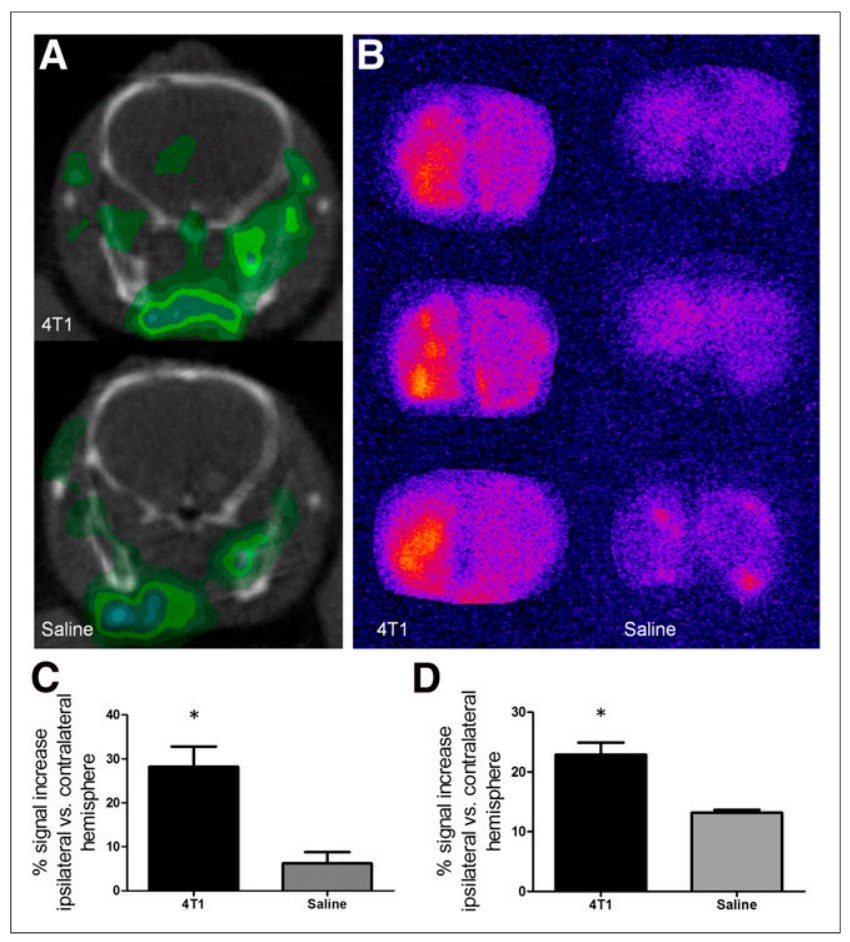

FIGURE 4. (A) Representative SPECT images at injection site (left hemisphere) from 4T1-GFP-injected mouse and saline-injected control. (B) ${ }^{123}$ |DPA713 binding was confirmed by autoradiography in 4T1-GFP-injected animals as compared with saline-injected animals. (C-D) Graphs show percentage signal increase in injected hemisphere, compared with control contralateral hemisphere for 4T1-GFP- $(n=6)$ and saline- $(n=3)$ injected animals for either SPECT (C) or autoradiography (D) data. ${ }^{*} P<0.05$.

However, by day 28, activation of both glial populations was comparable. These findings support the view that the intracerebral model represents a later time point in metastatic progression in which microglial and astrocyte responses are more evenly matched.

The development of techniques that enable earlier detection of brain metastases when therapy may be more beneficial is critical (3). In light of the robust glial response demonstrated here, we hypothesized that a marker of gliosis could provide a useful biomarker for metastatic burden. TSPO upregulation has been demonstrated in both astrocytes and microglia when they become reactive $(16,17)$, and numerous studies have demonstrated the use of radiolabeled agents targeting TSPO for the detection of neuropathology.

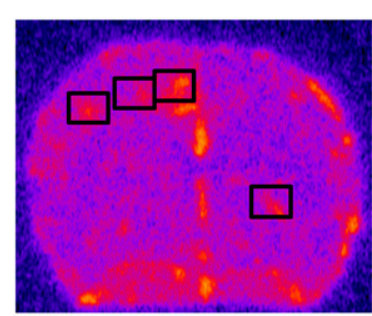

Autoradiography

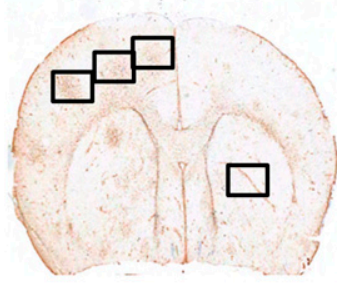

Immunohistochemistry

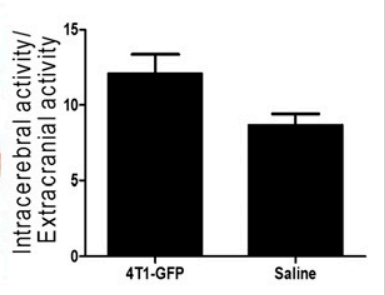

SPECT
FIGURE 5. Representative autoradiography image from mouse brain obtained $21 \mathrm{~d}$ after intracardiac injection of 4T1-GFP cells; areas of increased signal correlated spatially with immunohistochemical detection of tumor burden, as seen in immunohistochemistry image. Graph shows quantification of ${ }^{123}$-DPA713 binding (normalized activity), as determined by SPECT, in animals injected intracardially with either 4T1-GFP cells or saline.
Thus, our aim was to determine whether such a molecular imaging approach could provide a tool for the early detection of brain metastases. In accord with this hypothesis, increased TSPO expression was found on both astrocytes and microglia in the metastasis microenvironment in this study, as has been reported previously in other inflammatory conditions $(16,32)$. Interestingly, in the intracerebral model (day 14) TSPO upregulation appeared to be more robust in reactive astrocytes than activated microglia. Conversely, the reverse was found in the intracardiac model (day 21), with TSPO expression in astrocytes being largely restricted to the tumor periphery. These findings suggest that TSPO expression may reflect differential glial phenotypes and that induction of TSPO expression is context-dependent (33). Nevertheless, the combined TSPO signal from both glial populations supported the concept that radiolabeled compounds targeted to TSPO could be used to image metastasis-associated gliosis.

Initial TSPO imaging work was conducted in the intracerebral model, in which the tumor cells are injected into a known location within the left striatum. ${ }^{123}$ I-DPA713 accumulation was clearly evident at the site of tumor cell injection and to a significantly greater extent than was seen in saline-injected animals. Autoradiography provided higher-resolution confirmation of ${ }^{123}$ I-DPA713 binding in the tumor-bearing hemisphere. Spatial registration of the autoradiographic images with immunohistochemical staining demonstrated that radiotracer binding extended well beyond the area encompassing the metastases themselves and correlated spatially with the overall extent of astrocyte and microglial activation. However, the greatest intensity of compound binding appeared to correlate with the metastatic foci. The intense staining correlating with the tumor foci is likely due to the strong glial activation in this region, including dense microglial infiltration of the metastatic site and closely surrounding astrocyte activation.

Autoradiography data indicated that ${ }^{123}$ I-DPA713 also enabled detection of astrogliosis induced by metastases in the intracardiac model. However, it appears that the spatial resolution of SPECT imaging was insufficient to enable visualization of individual metastases at this time point in vivo, most likely because of their small size, compared with the metastatic foci in the intracerebral model $(\sim 100$ vs. $600-\mu \mathrm{m}$ diameter) and, hence, the associated area of gliosis ( $\sim 400$ vs. $\sim 1,800 \mu \mathrm{m})$.

Taken together, the intracerebral and intracardiac ${ }^{123}$ I-DPA713 SPECT data suggest a detection threshold for metastases lying between 100 and $600 \mu \mathrm{m}$ in diameter, with an associated area of astroglial activation of $0.120-120 \mathrm{~mm}^{2}$. Although the spatial resolution of SPECT (1-2 mm) will preclude detection of very small individual metastases and their associated glial response, this may be further compounded by limitations in contrast agent sensitivity. New-generation TSPO compounds labeled with ${ }^{18} \mathrm{~F}$ may enable a more sensitive detection of metastases from even earlier time points. Nevertheless, the limits of detection demonstrated here would still equate to considerably earlier detection than the current clinical approach of gadolinium-enhanced MR imaging (detection threshold, 0.5- to 1.0-cm tumor diameter).

Interestingly, recent work has suggested the potential for imaging breast cancer metastasis to the brain based on the TSPO expression by the breast cancer cells themselves (34-36). However, here we have dem- 
onstrated that this approach would target not only the metastatic cells themselves but also the glial response. This amplified region of detection will be of critical importance for lower-resolution clinical imaging methods. At the same time, our data indicate that such approaches cannot be considered to be specific to the tumor locus alone. Moreover, we found that the 4T1-GFP cells used in this study do not robustly express TSPO, in contrast to the metastatic human breast carcinoma cell line MDABR231, in which expression has previously been shown (37). These findings suggest that not all tumor cells will be TSPO-positive. In contrast, glial activation has been found to be consistent across several different types of brain metastasis. Taken together, the above data indicate the potential for earlier clinical detection of brain metastases through TSPO targeting of the metastasis-associated glial response.

\section{CONCLUSION}

We have demonstrated, in vivo, that the inflammatory tumor microenvironment can be targeted with current TSPO-targeted imaging agents to detect metastatic burden in the brain using the associated robust glial response. These findings suggest the potential for earlier clinical detection of brain metastases using TSPO-targeted agents, through either SPECT $\left({ }^{123} \mathrm{I}\right)$ or PET $\left({ }^{18} \mathrm{~F}\right)$ imaging, than is currently clinically possible.

\section{DISCLOSURE}

The costs of publication of this article were defrayed in part by the payment of page charges. Therefore, and solely to indicate this fact, this article is hereby marked "advertisement" in accordance with 18 USC section 1734. This work was funded by Cancer Research U.K., grant number C5255/A12678, and an MRC Centenary Early Career Award. No other potential conflict of interest relevant to this article was reported.

\section{ACKNOWLEDGMENT}

We thank Dr. Sean Smart for assistance with SPECT/CT.

\section{REFERENCES}

1. Nussbaum ES, Djalilian HR, Cho KH, Hall WA. Brain metastases: histology, multiplicity, surgery, and survival. Cancer. 1996;78:1781-1788.

2. Weil RJ, Palmieri DC, Bronder JL, Stark AM, Steeg PS. Breast cancer metastasis to the central nervous system. Am J Pathol. 2005;167:913-920.

3. Serres S, Soto MS, Hamilton A, et al. Molecular MRI enables early and sensitive detection of brain metastases. Proc Natl Acad Sci USA. 2012;109:6674-6679.

4. Zhang M, Olsson Y. Reactions of astrocytes and microglial cells around hematogenous metastases of the human brain: expression of endothelin-like immunoreactivity in reactive astrocytes and activation of microglial cells. J Neurol Sci. 1995;134:26-32.

5. He BP, Wang JJ, Zhang X, et al. Differential reactions of microglia to brain metastasis of lung cancer. Mol Med. 2006;12:161-170.

6. Mendes O, Kim HT, Lungu G, Stoica G. MMP2 role in breast cancer brain metastasis development and its regulation by TIMP2 and ERK1/2. Clin Exp Metastasis. 2007;24:341-351.

7. Mendes O, Kim HT, Stoica G. Expression of MMP2, MMP9 and MMP3 in breast cancer brain metastasis in a rat model. Clin Exp Metastasis. 2005;22:237-246.

8. Fitzgerald DP, Palmieri D, Hua E, et al. Reactive glia are recruited by highly proliferative brain metastases of breast cancer and promote tumor cell colonization. Clin Exp Metastasis. 2008;25:799-810.

9. Lorger M, Felding-Habermann B. Capturing changes in the brain microenvironment during initial steps of breast cancer brain metastasis. Am J Pathol. 2010; 176:2958-2971.

10. Samdani AF, Kuchner EB, Rhines L, et al. Astroglia induce cytotoxic effects on brain tumors via a nitric oxide-dependent pathway both in vitro and in vivo. Neurosurgery. 2004;54:1231-1237, discussion 1237-1238.

11. Neumann H, Medana IM, Bauer J, Lassmann H. Cytotoxic T lymphocytes in autoimmune and degenerative CNS diseases. Trends Neurosci. 2002;25:313-319.
12. Langley RR, Fan D, Guo L, et al. Generation of an immortalized astrocyte cell line from $\mathrm{H}-2 \mathrm{~Kb}$-tsA58 mice to study the role of astrocytes in brain metastasis. Int J Oncol. 2009;35:665-672.

13. Seike T, Fujita K, Yamakawa Y, et al. Interaction between lung cancer cells and astrocytes via specific inflammatory cytokines in the microenvironment of brain metastasis. Clin Exp Metastasis. 2011;28:13-25.

14. Xing F, Kobayashi A, Okuda H, et al. Reactive astrocytes promote the metastatic growth of breast cancer stem-like cells by activating Notch signalling in brain. EMBO Mol Med. 2013;5:384-396.

15. Squires RF, Brastrup C. Benzodiazepine receptors in rat brain. Nature. 1977; 266:732-734.

16. Cosenza-Nashat M, Zhao ML, Suh HS, et al. Expression of the translocator protein of $18 \mathrm{kDa}$ by microglia, macrophages and astrocytes based on immunohistochemical localization in abnormal human brain. Neuropathol Appl Neurobiol. 2009;35:306-328.

17. Lavisse S, Guillermier M, Herard AS, et al. Reactive astrocytes overexpress TSPO and are detected by TSPO positron emission tomography imaging. J Neurosci. 2012;32:10809-10818.

18. Ching AS, Kuhnast B, Damont A, Roeda D, Tavitian B, Dolle F. Current paradigm of the $18-\mathrm{kDa}$ translocator protein (TSPO) as a molecular target for PET imaging in neuroinflammation and neurodegenerative diseases. Insights Imaging. 2012;3:111-119.

19. Winkeler A, Boisgard R, Awde AR, et al. The translocator protein ligand $\left[{ }^{18} \mathrm{~F}\right]$ DPA-714 images glioma and activated microglia in vivo. Eur J Nucl Med Mol Imaging. 2012;39:811-823.

20. Tang D, Hight MR, McKinley ET, et al. Quantitative preclinical imaging of TSPO expression in glioma using N,N-diethyl-2-(2-(4-(2- ${ }^{18} \mathrm{~F}$-fluoroethoxy)phenyl)-5, 7-dimethylpyrazolo[1,5-a]pyrimi din-3-yl)acetamide. J Nucl Med. 2012;53:287-294.

21. Boutin H, Chauveau F, Thominiaux C, et al. ${ }^{11} \mathrm{C}-\mathrm{DPA}-713$ : a novel peripheral benzodiazepine receptor PET ligand for in vivo imaging of neuroinflammation. J Nucl Med. 2007;48:573-581.

22. Balathasan L, Beech JS, Muschel RJ. Ultrasonography-guided intracardiac injection: an improvement for quantitative brain colonization assays. Am J Pathol. 2013;183:26-34.

23. Reynolds A, Hanani R, Hibbs D, et al. Pyrazolo[1,5-a]pyrimidine acetamides: 4-Phenyl alkyl ether derivatives as potent ligands for the $18 \mathrm{kDa}$ translocator protein (TSPO). Bioorg Med Chem Lett. 2010;20:5799-5802.

24. Wang H, Pullambhatla M, Guilarte TR, Mease RC, Pomper MG. Synthesis of $\left[{ }^{125}\right.$ I] iodoDPA-713: a new probe for imaging inflammation. Biochem Biophys Res Commun. 2009;389:80-83.

25. Park ES, Kim SJ, Kim SW, et al. Cross-species hybridization of microarrays for studying tumor transcriptome of brain metastasis. Proc Natl Acad Sci USA. 2011;108:17456-17461.

26. Kim SJ, Kim JS, Park ES, et al. Astrocytes upregulate survival genes in tumor cells and induce protection from chemotherapy. Neoplasia. 2011;13:286-298.

27. Lin Q, Balasubramanian K, Fan D, et al. Reactive astrocytes protect melanoma cells from chemotherapy by sequestering intracellular calcium through gap junction communication channels. Neoplasia. 2010;12:748-754.

28. Amit M, Laider-Trejo L, Shalom V, Shabtay-Orbach A, Krelin Y, Gil Z. Characterization of the melanoma brain metastatic niche in mice and humans. Cancer Med. 2013;2:155-163.

29. Pukrop T, Dehghani F, Chuang HN, et al. Microglia promote colonization of brain tissue by breast cancer cells in a Wnt-dependent way. Glia. 2010;58:1477-1489.

30. Brantley EC, Guo L, Zhang C, et al. Nitric oxide-mediated tumoricidal activity of murine microglial cells. Transl Oncol. 2010;3:380-388.

31. Murata J, Ricciardi-Castagnoli P, Dessous L'Eglise Mange P, Martin F, JuilleratJeanneret L. Microglial cells induce cytotoxic effects toward colon carcinoma cells: measurement of tumor cytotoxicity with a gamma-glutamyl transpeptidase assay. Int J Cancer. 1997;70:169-174.

32. Harberts E, Datta D, Chen S, Wohler JE, Oh U, Jacobson S. Translocator protein $18 \mathrm{kDa}$ (TSPO) expression in multiple sclerosis patients. J Neuroimmune Pharmacol. 2013;8:51-57.

33. Batarseh A, Li J, Papadopoulos V. Protein kinase C epsilon regulation of translocator protein $(18 \mathrm{kDa})$ Tspo gene expression is mediated through a MAPK pathway targeting STAT3 and c-Jun transcription factors. Biochemistry. 2010;49:4766-4778.

34. Wyatt SK, Manning HC, Bai M, et al. Preclinical molecular imaging of the translocator protein (TSPO) in a metastases model based on breast cancer xenografts propagated in the murine brain. Curr Mol Med. 2012;12:458-466.

35. Wyatt SK, Manning HC, Bai M, et al. Molecular imaging of the translocator protein (TSPO) in a pre-clinical model of breast cancer. Mol Imaging Biol. 2010;12:349-358.

36. Vasdev N, Green DE, Vines DC, et al. Positron-emission tomography imaging of the TSPO with $\left[{ }^{18} \mathrm{~F}\right] \mathrm{FEPPA}$ in a preclinical breast cancer model. Cancer Biother Radiopharm. 2013;28:254-259.

37. Batarseh A, Barlow KD, Martinez-Arguelles DB, Papadopoulos V. Functional characterization of the human translocator protein $(18 \mathrm{kDa})$ gene promoter in human breast cancer cell lines. Biochim Biophys Acta. 2012;1819:38-56. 